\title{
Die Verbreitung des \\ Weiterlernens von \\ Lehrpersonen: Effekte der \\ Praxisgemeinschaft und des \\ Kompetenzselbstkonzeptes
}

\section{Doris Kunz Heim und Simone Rindlisbacher}

Das Weiterlernen von Lehrpersonen ist sowohl für die Schul- und Unterrichtsqualität als auch für die Umsetzung von Reformbestrebungen eine wichtige Voraussetzung. In der vorliegenden Studie wird der Einfluss von Faktoren des Schulkontextes und von personalen Faktoren der Lehrpersonen auf die Verbreitung des institutionellen und des informellen Weiterlernens untersucht. Anhand der Daten einer repräsentativen Stichprobe von Lehrpersonen der Sekundarstufe I der Kantone Aargau und Thurgau wird ein entsprechendes Modell geprüft. Die Ergebnisse zeigen, dass das informelle Weiterlernen durch die Ausprägung der Praxisgemeinschaft an der Schule, durch das Kompetenzselbstkonzept der Lehrpersonen und durch die Anzahl von Spezialfunktionen, welche eine Lehrperson an der Schule ausübt, beeinflusst wird. Entgegen den Erwartungen konnte das institutionelle Weiterlernen durch die postulierten Faktoren nicht erklärt werden.

\section{Einleitung}

Erziehungsdirektionen in der Schweiz haben in den letzten Jahren zahlreiche Reformbestrebungen für die Volksschule initiiert. Die Impulse zu diesen Bestrebungen stammen teilweise aus Quellen ausserhalb des Bildungssystems und betonen dabei verstärkt die Bereiche Management, Effizienz und Qualität (Brüsemeister \& Eubel, 2003). Lag zu Beginn des neuen Jahrtausends der Schwerpunkt auf neuen Leitungs- und Aufsichtsstrukturen, so zielen jüngere Reformbestrebungen auf Effizienz- und Qualitätsdimensionen, wie z.B. der frühere Einbezug des Fremdsprachenunterrichts oder der verbesserte Umgang mit Heterogenität.

Reformbestrebungen bewirken wenig, wenn sie nicht positiv erwünschte Folgen für die Schule und den Unterricht haben (Fichten, 2007). Dass jedoch viele Reformbestrebungen aufgrund mangelnder Umsetzungstreue nicht die beabsichtigten Folgen zeitigen, ist in der Literatur breit belegt (Brüsemeister \& Eubel, 2003; Clarke \& Hollingsworth, 2002; Ghisla, 1999; McLaughlin, 1998; Ri- 
chardson \& Placier, 2001; Rolff, 1988, 1995). Die Umsetzung von Reformbestrebungen im Unterricht bedingt ein Um- und Weiterlernen durch die Lehrpersonen (Fichten, 2007). Das Weiterlernen ist zudem erforderlich, um die Unterrichts- und Schulqualität zu erhalten und zu erhöhen. Während dem Prozesse der Schulentwicklung im Rahmen der Bildungsforschung eine breite Aufmerksamkeit erfahren haben, blieb das Weiterlernen von Lehrpersonen im deutschsprachigen Raum bisher wenig erforscht.

In diesem Beitrag werden Faktoren aufgezeigt, die das Weiterlernen von Lehrpersonen positiv beeinflussen. Es handelt sich dabei um Einflüsse des Schulkontextes und um personale Faktoren der Lehrpersonen. Nebst dem institutionellen Weiterlernen im Rahmen der herkömmlichen Weiterbildungsgefässe wird in diesem Beitrag erstmals dem informellen Lernen im Rahmen des beruflichen Alltags vertieft Beachtung geschenkt. Anschliessend wird ein Wirkungsmodell der als relevant identifizierten Faktoren geprüft. Die Untersuchung beschränkt sich auf die quantitative Komponente der beiden Formen des Weiterlernens, d.h. es wird untersucht, wie oft Lehrpersonen Lerngelegenheiten wahrnehmen. Aspekte des Inhalts sowie der Wirkung derselben auf die Unterrichts- und Schulqualität werden nicht berücksichtigt.

Angloamerikanische Studien zum Weiterlernen von Lehrpersonen werden häufig im Zusammenhang mit der Umsetzung einer bestimmten Reformbestrebung durchgeführt und liegen in der Form von Fallstudien vor (Richardson \& Placier, 2001). Der vorliegende Beitrag stützt sich auf die Ergebnisse der Analyse von Daten eines repräsentativen Samples von 812 Lehrpersonen der Sekundarstufe I der Kantone Aargau und Thurgau, die unabhängig von einer spezifischen Reformbestrebung erhoben worden sind.

\section{Institutionelles und informelles Weiterlernen}

Die Verbreitung des Weiterlernens von Lehrpersonen wird definiert als die Häufigkeit der Teilnahme an beruflichen Lernhandlungen (Kwakman, 2003). Dabei wird wie erwähnt zwischen institutionellem und informellem Weiterlernen unterschieden. Institutionelles Weiterlernen erfolgt im Rahmen herkömmlicher Formen der Lehrpersonenweiterbildung, bei denen die Lernanlässe durch Dritte, also nicht durch die Lehrpersonen selbst, organisiert und durch Experten oder Expertinnen geleitet werden. Die Lernanlässe des informellen Lernens hingegen werden von den beteiligten Lehrpersonen selbst organisiert und geleitet.

Die Verbreitung des institutionellen Weiterlernens wird üblicherweise mit der Anzahl absolvierter Weiterbildungstage pro Zeiteinheit, meistens pro Schuljahr, erfasst. Für die Erhebung des informellen Weiterlernens schlägt Kwakman (2003) die faktoranalytisch überprüfte Operationalisierung der drei Dimensionen instruktionelle, individuelle und kollaborative Aktivitäten vor (instructional, individual and collaborative activities) und erfasst deren Verbreitung mit Hilfe einer Selbsteinschätzung der Lehrpersonen auf einer vier-Punkte Skala. 
Im Rahmen der jüngsten Erhebung über die Verbreitung des institutionellen Weiterlernens in der Schweiz stellt Landert (1999) fest, dass 40\% der befragten Schweizer Lehrpersonen das Weiterbildungsangebot ungenügend nutzen, d.h. sie absolvieren weniger als fünf Weiterbildungstage pro Jahr (ebd., S.181). Der Autor geht davon aus, dass 20-30\% der Lehrerschaft von den institutionellen Anbietern für Weiterbildung nicht erreicht werden und 10-20\% höchstens sporadische Nutzer und Nutzerinnen sind.

Das informelle Weiterlernen hat in den letzten Jahren an Aufmerksamkeit gewonnen, weil von ihm eine bessere Wirkung in Bezug auf die Umsetzung von Reformbestrebungen erhofft wird, nachdem die Grenzen des institutionellen Weiterlernens diesbezüglich deutlich geworden sind. Die begrenzte Wirkung des institutionellen Weiterlernens wird hauptsächlich auf zwei Ursachen zurückgeführt: Erstens werden die von Expert/innen vorgestellten neuen Unterrichtspraktiken nur dann übernommen, wenn diese mit den Einstellungen und Werthaltungen der Lehrpersonen bezüglich des Unterrichtens kompatibel sind. Zweitens wird die Umsetzung von Neuerungen durch die höhere Priorität von Pendenzen aus dem Alltag behindert, wenn der längerfristige Support für die Umsetzung fehlt (Kunz Heim, 2002; Landert, 1999; Mutzeck, 1992; Richardson \& Placier, 2001; Rudduck, 1988; Rüegg, 2000).

$\mathrm{Ob}$ das informelle Weiterlernen tatsächlich eine bessere Wirkungen auf die Umsetzung von Reformbestrebungen hat, muss sich im Rahmen von künftigen Studien weisen. Das informelle Weiterlernen ist zwar in den Kontext der Alltagspraxis eingebettet, so dass durch die Steuerung der beteiligten Personen relevante Fragen und entstehende Dissonanzen zu bisherigen Einstellungen unmittelbar bearbeitet werden können (Kwakman, 2003). Es ist aber zu vermuten, dass auch das informelle Lernen seine Grenzen hat, z.B. durch die höhere Priorität durch Pendenzen aus dem Alltag.

\section{Der Einfluss des Schulkontextes: Schulkultur und Praxisgemeinschaft}

Der Schulkontext und dabei insbesondere die Schulkultur und die Praxisgemeinschaft sind in der Literatur als Faktoren identifiziert worden, die das Weiterlernen von Lehrpersonen beeinflussen. Die Schulkultur wird definiert als ein Aggregat von Meinungen, Einstellungen und Werthaltungen des Kollegiums, das implizit von allen geteilt und als gültig anerkannt wird. Dieser unausgesprochene Konsens bestimmt das Handeln der Lehrpersonen und somit auch ihr Weiterlernen in hohem Masse (Fend, 1998; Hargreaves, 1997). Bereits seit den 80er Jahren des letzten Jahrhunderts betonen die Autorinnen und Autoren, dass die Schulkultur nicht nur positive Effekte auf das Weiterlernen von Lehrpersonen hat. Genauso gut kann sie jede Innovation unterbinden. Rosenholtz, 
Bassler und Hoover-Dempsey (1986) sprechen diesbezüglich von learning enriched oder von learning impoverished schools, McLaughlin (1993) von kollegialen oder isolierenden Schulen und Fend (1998) von einem produktiven, einem distanzierten oder einem unproduktiven Modus der Schulkultur.

Nicht nur die Einzelschule als Ganzes beeinflusst das Weiterlernen, sondern auch die Zusammenarbeit in kleineren Einheiten wie z.B. Abteilungen, Fachschaften oder Teams von Lehrpersonen, im Folgenden Praxisgemeinschaften genannt. Deutlich aufgezeigt wurde dies durch die Studie von McLaughlin (1993). Die Autorin identifizierte drei unterschiedliche Reaktionsformen von Lehrpersonen auf Herausforderungen, welche durch den Wandel des sozioökonomischen Hintergrundes ihrer Schülerinnen und Schüler entstanden waren (z.B. zunehmende Gewaltbereitschaft, fehlende Kenntnisse der Standardsprache oder Herkunft aus bildungsfernen Familien). Zwei disfunktionale Reaktionen der Lehrpersonen bestanden entweder darin, die herkömmlichen Standards und Unterrichtsformen aufrecht zu erhalten oder die Leistungserwartungen zu senken. Die funktionale Reaktion, welche die Lernchancen dieser Schülerinnen und Schüler intakt hielt, bestand in der Aufrechterhaltung der Leistungserwartungen bei gleichzeitiger Anpassung der Unterrichtsgestaltung. McLaughlin (1993) zeigte auf, dass die Reaktion der Lehrpersonen von deren Berufsverständnis und dem kollegialen Umfeld abhing. Funktionale Reaktionen zeigten sich häufiger in Abteilungen, wo Lehrpersonen Unterricht gemeinsam planten, sich gegenseitig unterstützten und sich über ihre Erfahrungen austauschten. Als Bezeichnung für solche Arbeitsteams hat sich in den letzten zehn Jahren auch der Begriff der Professionellen Lerngemeinschaften etabliert.

\section{Multifaktorielle Erklärungsansätze für das Weiterlernen von Lehrpersonen}

Mehrere Arbeiten weisen einen multifaktoriellen Ansatz auf und beleuchten nebst den personalen Merkmalen, der Schulkultur und der Praxisgemeinschaft auch den Einfluss weiterer Merkmale des Schulkontextes auf das Weiterlernen von Lehrpersonen.

Smylie (1988) fand im Zusammenhang mit einem freiwilligen Weiterbildungsprogramm zwei Faktoren, die auf die Umsetzung der Inhalte durch die Lehrpersonen wirken: Den Grad der Selbstwirksamkeitsüberzeugung der Lehrpersonen und die Grösse der Klasse, welche diese zum Zeitpunkt des Weiterbildungskurses unterrichteten und zwar setzten Lehrpersonen mit grösseren Klassen entgegen den Erwartungen des Autors die Neuerung eher um.

Was die informellen Formen des Weiterlernens betrifft, so identifizierten Rosenholtz et al. (1986) im Rahmen eines Modelltests folgende positiven Einflussfaktoren: Ein kollegiales Führungsverständnis des Schulleiters, eine sorgfältige Auswahl bei der Anstellung neuer Lehrpersonen, die Zielorientierung der Akti- 
vitäten innerhalb des Kollegiums, die Evaluation der Lehrerarbeit durch Schulleitende, Zusammenarbeit unter den Lehrpersonen, gemeinsame Anstrengungen aller Lehrpersonen in Bezug auf die Disziplinerhaltung an der Schule und die Koordination der Unterrichtsinhalte unter den Lehrpersonen mit dem Ziel der Kontinuität innerhalb eines Klassenzuges.

Richardson und Placier (2001) extrahierten aus ihrer Review folgende vier Einflussfaktoren auf informelle Formen des Weiterlernens von Lehrpersonen: der schulische Kontext, in dem sich die Lehrperson befindet, die eigenen Unterrichtsphilosophien, die Selbstwirksamkeitsüberzeugung sowie die Ansicht, inwieweit Kompetenzen des Lehrerhandelns erlernbar oder eine Funktion der Begabung sind.

Kwakman (2003) fand den stärksten Einfluss auf das Weiterlernen in Faktoren der persönlichen Einstellung, wie dem professionellen Engagement der Lehrpersonen. Ebenfalls wichtig war der geschätzte Grad der Praktikabilität und der Sinnhaftigkeit der Neuerung. Deutlich negativ korrelierte ein wahrgenommener Kontrollverlust in Bezug auf die eigene Arbeit. Schwach positiv korrelierten die Einflussfaktoren des Arbeitsplatzes, wie vorhandene Lernanregungen durch die Schule, wahrgenommene Vielseitigkeit der Arbeit und Mitsprachemöglichkeiten in Bezug auf das Schulgeschehen.

Zusammenfassend kann festgehalten werden, dass sowohl kontextuelle Faktoren der Schule als auch personale Faktoren der Lehrpersonen das Weiterlernen beeinflussen.

\section{Exkurs: Selbstwirksamkeit und Kompetenzselbst- konzept}

Wie erwähnt haben verschiedene Autoren den Einfluss der Selbstwirksamkeitsüberzeugung (self efficacy) auf das Weiterlernen von Lehrpersonen nachgewiesen (Richardson \& Placier 2001; Smylie, 1988). In der vorliegenden Studie interessierte, ob an ihrer Stelle Effekte des Konstruktes des Kompetenzselbstkonzeptes (self-concept beliefs) auf das Weiterlernen nachgewiesen werden können.

Die beiden Konstrukte weisen Gemeinsamkeiten in Bezug auf ihre Effekte aber Unterschiede bezüglich der Grundausrichtung und der Operationalisierung auf. Es sind beides Komponenten der Selbstkognition (Pajares \& Schunk, 2001) oder der Selbstrepräsentation (Skaalvik, 1997) und beide zeigen Effekte auf die Lernleistungen (academic achievement) von Individuen (Pajares \& Schunk, 2001). Die Selbstwirksamkeitsüberzeugung ist die Einschätzung des Vertrauens, das jemand in den erfolgreichen Einsatz seiner Fähigkeiten hat und bezieht sich somit vor allem auf die Einschätzung, ob eine bestimmte Aufgabe bewältigt werden kann oder nicht. Die Selbstwirksamkeitsüberzeugung resultiert aus den Erfahrungen, die das Individuum bei der Bewältigung von Aufgaben gemacht hat 
(ebd., S. 243). Das Selbstkonzept ist eine Beschreibung des Selbst, die auch eine bewertende Komponente beinhaltet und sich auf die Einschätzung bezieht, wie gut ein Individuum seine Fähigkeiten in einem bestimmten Bereich einschätzt oder wie gut es sich in diesem Bereich fühlt (ebd., S. 245). Das Selbstkonzept beruht auf einem Vergleich mit Fähigkeiten und Merkmalen anderer. Welche Bereiche das Individuum für die Etablierung des Selbstkonzeptes berücksichtigt, hängt von deren individuellen Bedeutung ab. So kann ein Individuum in einem bestimmten Bereich, z.B. im Sport, über eine grosse Selbstwirksamkeitsüberzeugung verfügen: Diese trägt jedoch nur dann positiv zum Selbstkonzept bei, wenn das Individuum diesen Bereich als wichtig erachtet.

Beide Konstrukte sind bereichsspezifisch und sollten gemäss den Autoren auch so operationalisiert werden. Die nachgewiesenen Effekte des generellen Selbstkonzeptes auf die Lernleistung sind gering, in einigen Studien sogar negativ. Wird das Selbstkonzept bereichsspezifisch hinsichtlich spezifischer Kompetenzen erfasst, z.B. das Selbstkonzept in Naturwissenschaften, können hohe Relationen mit den entsprechenden Lernleistungen nachgewiesen werden (ebd., S. 247).

\section{Fragestellung}

Die vorliegende Studie weist die vier folgenden Fragestellungen auf:

1) Wie verbreitet ist das institutionelle und das informelle Weiterlernen?

2) Welche personalen Faktoren und welche kontextuellen Faktoren der Schule beeinflussen die Verbreitung des Weiterlernens von Lehrpersonen? - Als Prädiktorvariablen des Schulkontextes wurden die Ausprägung der wahrgenommenen Führung der Schulleitung sowie die Ausprägung der Praxisgemeinschaft bestimmt (Abbildung 1). Prädiktorvariable auf der personalen Ebene ist das Kompetenzselbstkonzept.

3) Welche berufsbezogenen Merkmale beeinflussen das Weiterlernen von Lehrpersonen? - Was diese Merkmale betrifft, so sind das Pensum und die Anzahl schulischer Spezialfunktionen hervorzuheben. So fand bereits Landert (1999), dass Lehrpersonen mit einer vergleichsweise hohen Nutzung des institutionellen Weiterbildungsangebotes über ein hohes Pensum verfügen.

4) Wie wirken die ausgewählten Faktoren zusammen? - In Bezug auf diese Fragestellung erschwerte das Fehlen empirisch abgestützter Modelle die Bestimmung der relevanten Hypothesen stark. Erwartet wurde, dass die wahrgenommene Führung und das Kompetenzselbstkonzept je einen direkten Einfluss auf die Verbreitung des Weiterlernens zeigen (Abbildung 1). Von der Praxisgemeinschaft wurde erwartet, dass diese nicht nur einen direkten Einfluss auf das Weiterlernen von Lehrpersonen hat, sondern auch durch das Kompetenzselbstkonzept mediierte Effekte zeigen sollte. Letztere Annahme beruht auf der Feststellung von Pajares und Schunk (2001), wonach das Kompetenzselbstkonzept u.a. auf dem Vergleich mit Fähigkeiten und Merk- 
malen anderer beruht. Die Praxisgemeinschaft kann z.B. dann positive Effekte auf das Kompetenzselbstkonzept haben, wenn Lehrpersonen feststellen, dass sie in einem kompetenten Team mithalten können.

In Bezug auf die soziodemografischen Variablen wurden von der Höhe des Pensums sowie der Anzahl der Spezialfunktionen, die eine Lehrperson ausübt, Effekte auf das Weiterlernen erwartet. Erwartet wurden vermehrte Lernaktivitäten bei Lehrpersonen mit höherem Pensum und mehr Spezialfunktionen. Bezüglich der Anzahl Spezialfunktionen wurde ein direkter Effekt auf das Weiterlernen erwartet. Die Annahme lautete, dass Lehrpersonen, die eine oder mehrere Spezialfunktionen übernehmen, ein hohes Engagement für die Schule aufweisen und dieses Engagement auch im Bereich des Weiterlernens zeigen. Zudem scheint es auch wahrscheinlich, dass entsprechende Spezialfunktionen das Kompetenzselbstkonzept von Lehrpersonen positiv beeinflussen.

\section{Schulkontext Weiterlernen}

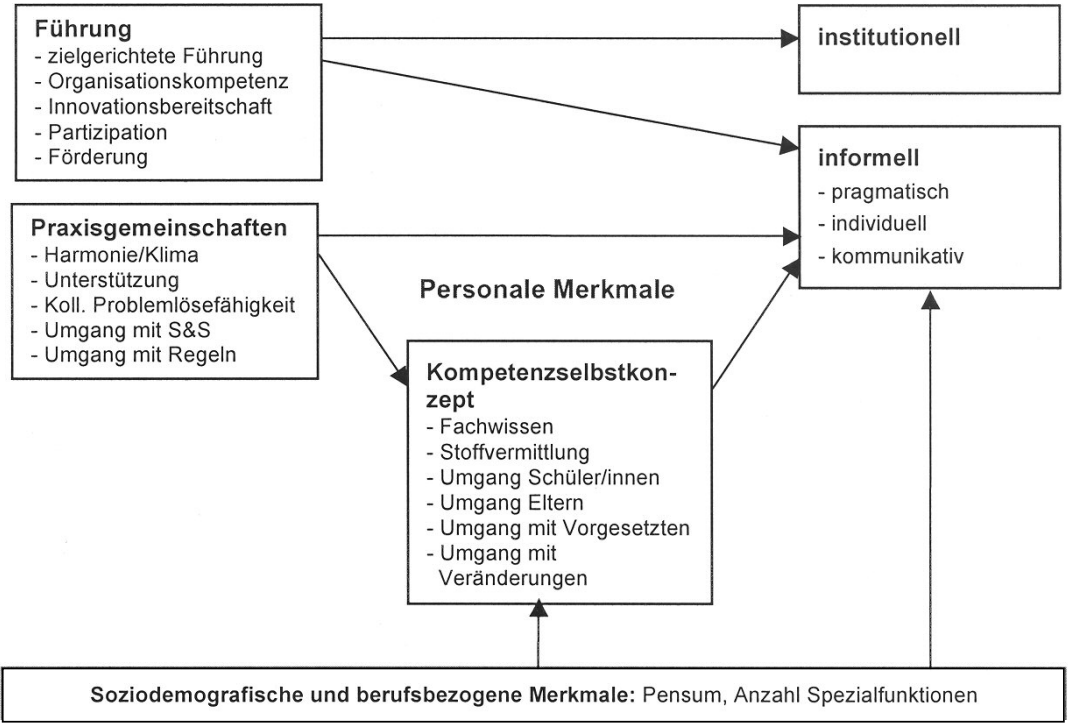

Abbildung 1: Heuristisches Modell

\section{Methode}

Design

Die Datenerhebung erfolgte im Herbst 2005 anhand einer standardisierten, schriftlichen und anonymen Querschnittbefragung von Lehrpersonen der Se- 
kundarstufe I der Kantone Aargau und Thurgau. Die Adressen konnten aus den Adressdateien der Bildungsverwaltungen gezogen werden.

Wie in Tabelle 1 ersichtlich sind die Daten aus dem Aargau sowohl in Bezug auf den Rücklauf (50.9\%), als auch in Bezug auf die Repräsentativitätsmerkmale unproblematisch. Im Kanton Thurgau wurde hingegen (wahrscheinlich aufgrund einer zu grossen Befragungsdichte im Herbst 2005) lediglich ein genügender Rücklauf (28.3\%) erzielt. Es kann daher vermutet werden, dass insbesondere unter den Lehrpersonen des Thurgaus aber damit auch in den Daten insgesamt, die an Weiterbildungsfragen stärker interessierten Lehrpersonen eher überrepräsentiert sind.

Tabelle 1: Kennwerte der Stichprobe und Rücklauf

\begin{tabular}{|l|l|c|c|c|}
\hline & & Grundgesamtheit & Stichprobe & Rücklauf \\
\hline \multirow{3}{*}{ AG } & $\mathrm{N}$ & 3625 & 1150 & $585(50.9 \%)$ \\
\cline { 2 - 5 } & Frauen & $58.6 \%$ & $56.3 \%$ & $57.9 \%$ \\
\cline { 2 - 5 } & Altersdurchschnitt & $43.6 \mathrm{Jahre}$ & $43.1 \mathrm{Jahre}$ & $43.2 \mathrm{Jahre}$ \\
\hline \multirow{3}{*}{ TG } & $\mathrm{N}$ & 1068 & 841 & $227(28.3 \%)$ \\
\cline { 2 - 5 } & Frauen & $47.9 \%$ & $47.3 \%$ & $47.6 \%$ \\
\cline { 2 - 5 } & Altersdurchschnitt & $41.7 \mathrm{Jahre}$ & keine Angabe & $43.2 \mathrm{Jahre}$ \\
\hline \multicolumn{2}{|l|}{ Total } & $\mathrm{N}=\mathbf{4 6 9 3}$ & $\mathrm{N}=\mathbf{1 9 5 3}$ & $\mathrm{N}=\mathbf{8 1 2}(\mathbf{4 1 . 6 \% )}$ \\
\hline
\end{tabular}

\section{Instrumente}

\section{Kriteriumsvariablen}

Das institutionelle Weiterlernen wurde in Bezug auf Landert (1999) mit der Anzahl der absolvierten Weiterbildungstage im Zeitraum der drei Schuljahre vor dem Befragungszeitpunkt erhoben (schulinterne, kantonale, vom Inspektorat, von der Schulaufsicht oder durch Schule und Weiterbildung Schweiz SWCH organisierte Weiterbildung; sowie Supervision, Coaching oder Beratung).

Für das informelle Weiterlernen wurden die drei Subskalen kommunikative Aktivitäten (collaborative activities), individuelle Aktivitäten (individual activities) und pragmatische Aktivitäten (instructional activities) von Kwakman (2003) übernommen und ins Deutsche übersetzt, wobei die Skalentitel leicht verändert wurden. Kommunikative Aktivitäten umfassen den Austausch von Erfahrungen in Bezug auf die Berufstätigkeit («mit Kolleg/innen überlegen, wie einzelne Schüler/innen besser gefördert werden könnten»), individuelle Aktivitäten sind solche, die alleine ausgeführt werden («Rückmeldungen von Schüler/innen einholen») und pragmatische Aktivitäten sind solche, die auf den Unterricht ausge- 
richtet sind und mit Kolleginnen kooperativ ausgeführt werden («mit Kolleg/innen den Unterricht vorbereiten»). Da die Skalierung von Kwakman ( 1 = hardly ever; 2 = sometimes; 3 = fairly often; 4 = often) nur sehr vage Angaben über die Verbreitung des informellen Weiterlernens zulässt und unklar bleibt welche Referenz die Antwortenden implizieren (häufig im Vergleich zu anderen Tätigkeiten, selten im Vergleich zu anderen Lehrpersonen etc.) wurde sie für die vorliegende Studie erweitert und präzisiert und eine 7-stufigen Skala ( 1 =fast nie/nie bis $7=$ mehrmals pro Woche) verwendet.

\section{Prädiktorvariablen}

Die wahrgenommene Führung wurde mit fünf Subskalen erfasst, wovon vier von Bonsen, Van der Gathen, Iglhaut \& Pfeiffer (2002) stammen und auf Krüger (1994) resp. Fend (1998) zurück gehen. Es handelt sich um die Skalen zielgerichtete Führung («Die Schulleitung arbeitet an der Entwicklung von langfristigen Zielen für die Schule»), Organisationskompetenz («Die Schulleitung besitzt immer einen genauen Überblick darüber, was an der Schule vorgeht»), Innovationsbereitschaft («Die Schulleitung ist neuen Ideen und Entwicklungen der pädagogischen Diskussion und Forschung gegenüber stets aufgeschlossen») und Partizipation in der Entscheidungsfindung («Die Schulleitung entscheidet häufig über die Köpfe des Kollegiums hinweg»). Die fünfte Subskala, principal leadership for reform, wurde am Center for Research on the Context of Teaching (CRC, 2001) in Stanford, USA, entwickelt, ins Deutsche übersetzt und mit dem Titel Förderung versehen («Die Schulleitung schafft Gelegenheit für den Erfahrungsaustausch»). Die Antworten zu allen Items wurden mittels einer 4-stufigen Skala erfasst ( $1=$ trifft völlig zu bis $4=$ trifft nicht $\mathrm{zu}$ ).

Für die Erhebung der Ausprägung der Praxisgemeinschaft wurden wiederum fünf Subskalen verwendet. Die erste, Harmonie/Klima im Kollegium, stammt aus dem Survey-Feedback-Instrument zur Organisationsdiagnose ( An meiner Schule gibt es Gruppen im Kollegium, die nichts miteinander zu tun haben wollen») (Ulber, 1998). Drei weitere ins Deutsche übersetzte Subskalen stammen vom Center for Research on the Context of Teaching (CRC, 2001). Es handelt sich um die Subskalen Unterstützung («An meiner Schule spornen sich die Kolleg/innen gegenseitig an, noch besser zu werden»), kollektive Problemlösefähigkeit («An meiner Schule werden Probleme gelöst, es wird nicht nur darüber gesprochen») sowie Umgang mit Schülerinnen und Schülern (Commitment) («An meiner Schule begegnen die Lehrpersonen den Schüler/innen mit Wertschätzung»). Die fünfte, ins Deutsche übersetzte Subskala, Umgang mit Regeln, stammt von Rosenholtz (1991) («An dieser Schule beteiligen sich die Lehrpersonen an der Festlegung der Regeln»). Die Antworten zu allen Items wurden ebenfalls mittels einer 4-stufigen Skala erfasst ( $1=$ stimmt genau bis $4=$ stimmt nicht). - Währenddem die ersten beiden Skalen (Hamonie/Klima; Unterstützung) Aspekte der Kooperation betreffen, so beziehen sich die anderen drei (kollektive 
Problemlösefähigkeit; Umgang mit Schüler/innen; Umgang mit Regeln) auf Zielsetzungen und gemeinsame Werthaltungen. Dies entspricht zwei von drei Postulaten von Bonsen und Rolff (2006), welche einen Klärungsbedarf in Bezug auf den Terminus der Professionellen Lerngemeinschaften identifizieren und zum Schluss kommen, dass nebst der Kooperation das Vorhandensein von pädagogischen Normen und Zielen sowie die Überwindung des Unterrichts in privater Isolation wichtige Komponenten von Professionellen Lerngemeinschaften sind.

Das Kompetenzselbstkonzept wurde mit Hilfe von vier eigens entwickelten Subskalen erhoben. Die Subskalen erfassen die Dimensionen Fachwissen («Fachlich auf dem neuesten Stand bleiben»), Stoffvermittlung («Unterschiedliche Lernaufgaben entwickeln, die den individuellen Möglichkeiten der Schüler/innen Rechnung tragen»), soziale Kompetenz im Umgang mit Schüler/innen («Auch schwierige Schüler/innen in die Klasse integrieren können»), soziale Kompetenz im Umgang mit Eltern («Mit Eltern aus anderen Kulturen gut zusammenarbeiten»), soziale Kompetenz im Umgang mit Vorgesetzten («Unstimmigkeiten mit dem/der Vorgesetzten klären») sowie Umgang mit Neuerungen («Freude daran haben, Neues auszuprobieren»). Die Antwortvorgabe war 4-stufig ( $1=$ weniger kompetent bis $4=$ sehr kompetent).

Bei der Anzabl Spezialfunktionen handelt es sich um verschiedene Aufgaben, die für das Funktionieren der Schule oder deren Repräsentation gegen aussen notwendig sind und von Lehrpersonen übernommen werden, wie z.B. die administrative Funktion des Erstellens des Gesamtstundenplans für die Schule, die pädagogische Funktion eines oder einer Sachverständigen für Gesundheitsförderung oder die Mitarbeit in der Arbeitsgruppe für das nächste Jugendfest. Das Pensum wurde in Form der Höhe der Stellenprozente resp. der Wochenlektionen erhoben.

\section{Anmerkung zu den statistischen Verfahren}

Um den Indikator für institutionelles Weiterlernen bilden zu können, sollten die verschiedenen Angaben der interessierenden Weiterbildungsformen summiert werden. Für die wenigen Ausreisser mit sehr hohen Werten konnten in fast allen Fällen plausible Erklärungen gefunden werden (Verwechslung der Kategorien bzw. Einbezug der Anzahl Tage Grundausbildung). Trotzdem konnte die (rechtsschiefe) Antwortverteilung aufgrund der grossen Häufigkeiten im Bereich zwischen 0 und 5 Tagen $(22.5 \%)$ und der relativ weiten Streuung nicht als näherungsweise normalverteilt bezeichnet werden. Daher wurde der Indikator des institutionellen Lernens für die inferenzstatistischen Analysen logarithmiert (Tabachnick \& Fidell, 1989, S. 82f.). Der transformierte Indikator korreliert mit der originalen Variable auf dem Niveau $r=.84$. 
Die zweite Kriteriumsvariable informelles Weiterlernen wurde vor der Skalenbildung z-standardisiert, um die unterschiedlichen Distanzen zwischen den Kategorien auszugleichen.

Alle latenten Konstrukte, also das informelle Weiterlernen, die Praxisgemeinschaft und das Kompetenzselbstkonzept wurden den üblichen Verfahren der Evaluation und Interpretation von Item- und Skaleneigenschaften unterzogen, sowie anhand einer konfirmatorischen Faktorenanalyse (CFA) validiert (Kunz Heim, Trachsler, Rindlisbacher \& Nido, 2007). Mittels eines Strukturgleichungsmodells wurde das Zusammenspiel kontexueller und personaler Prädiktoren analysiert. Sämtliche Transformationen und Analysen wurden mit SPSS 15 und Amos 16 durchgeführt.

\section{Ergebnisse}

\section{Die Verbreitung des institutionellen Weiterlernens}

Insgesamt beträgt die Anzahl absolvierter Weiterbildungstage im Beobachtungszeitraum der letzten drei Jahre vor dem Befragungszeitpunkt 15 Tage $(\mathrm{N}=768)$. Die Häufigkeit der Anzahl Weiterbildungstage ist so verteilt, dass viele Lehrpersonen die institutionellen Weiterbildungsangebote nur wenig nutzen und wenige Lehrpersonen dafür viel Weiterbildung vermelden: 64\% der Lehrpersonen geben an, die institutionellen Angebote in den letzten drei Jahren weniger als 5 Tage pro Jahr belegt zu haben (davon 6.6\% gar nicht), während $31.5 \%$ zwischen 5 bis 9 Weiterbildungstage nennen. Die restlichen $4.5 \%$ geben an, pro Jahr zwischen 10 bis 24 Weiterbildungstage absolviert zu haben.

\section{Verbreitung des informellen weiterlernens}

Was die informelle Komponente des Weiterlernens betrifft, konnten die drei Subdimensionen, die bereits Kwakman (2003) unterschied, in der konfirmatorischen Faktorenanalyse (CFA) bestätigt werden, obwohl die Antwortvorgaben in der vorliegenden Studie wie erwähnt mit konkreteren Zeitbezügen operationalisiert wurden. Inhaltlich erhielten die Subdimensionen jedoch leicht andere Akzente, was auch eine Anpassung zweier Skalentitel nahelegte. Die so genannt pragmatischen Aktivitäten (instructional activities) umfassen den Austausch von Unterrichtsmaterialien, eine gemeinsame Unterrichtsvorbereitung sowie verbindliche Absprachen bezüglich des Unterrichtens. Da jede Lehrperson ihre eigene Herangehensweise an Unterrichtsmaterialien oder an die Unterrichtsplanung hat, sind in dieser Subdimension Aktivitäten zusammengefasst, die voraussetzen, dass die Lehrpersonen darauf verzichten, exakt ihre Sichtweise umzusetzen und darauf vertrauen, dass auch die Herangehensweise des Partners oder der Partnerin erfolgversprechend ist. Gleichzeitig sind sie im Vergleich zu 
den kommunikativen Aktivitäten deutlicher handlungs- und anwendungsbezogen. Bei der zweiten Form, den so genannt individuellen Aktivitäten (individual activities) wird vor allem im Alleingang gelernt, indem zum Beispiel Rückmeldungen von Schülerinnen und Schülern eingeholt, Fachliteratur gelesen und neue Unterrichtsformen ausprobiert werden. Bei der dritten Form, den kommunikativen Aktivitäten (collective activities), tauschen sich Lehrpersonen über den Unterricht aus, überlegen gemeinsam, wie Schülerinnen und Schüler besser gefördert oder wie auftauchende Probleme im Unterricht gelöst werden können.

Tabelle 2 zeigt die Zuordnungen der Items zu den Subdimensionen mit den entsprechenden standardisierten Faktorladungen (FL). In der letzten Spalte sind die Mittelwerte zur Frequenz der Aktivitäten aufgeführt. Die meisten Lernaktivitäten werden zwischen mehrmals pro Jahr $(=3)$ und mehrmals pro Monat $(=5)$ ausgeführt.

\section{Tabelle 2: Dimensionen und Mittelwerte der Aktivitäten des informellen Lernens}

\begin{tabular}{|c|c|c|c|c|}
\hline & Subkonstrukt & $\mathrm{FL}^{\mathrm{a}}$ & $M^{b}$ & SD \\
\hline $\begin{array}{l}\text { Mit Kolleg/innen im Hinblick auf das Unterrichten } \\
\text { gegenseitig verbindliche Absprachen treffen. }\end{array}$ & Pragmatische & .78 & 3.8 & 1.7 \\
\hline Mit Kolleg/innen den Unterricht vorbereiten & Aktivitäten & .74 & 3.0 & 1.7 \\
\hline Unterrichtsmaterial von Kolleg/innen übernehmen & & .72 & 3.4 & 1.5 \\
\hline Neue Unterrichtsformen ausprobieren. & & .69 & 3.3 & 1.2 \\
\hline Rückmeldungen von Schüler/innen einholen. & Individuelle & .57 & 3.4 & 1.3 \\
\hline $\begin{array}{l}\text { Pädagogisch-didaktische Bücher oder Zeitschriften } \\
\text { lesen. }\end{array}$ & Aktivitäten & .56 & 3.2 & 1.5 \\
\hline Fachbezogene Bücher oder Zeitschriften lesen. & & .50 & 4.5 & 1.5 \\
\hline $\begin{array}{l}\text { Kollegen/innen unterstützen, die Probleme im } \\
\text { Unterricht haben. }\end{array}$ & & .75 & 3.4 & 1.6 \\
\hline $\begin{array}{l}\text { Mit Kolleg. überlegen, wie einzelne Schüler/innen } \\
\text { besser gefördert werden könnten. }\end{array}$ & $\begin{array}{l}\text { Kommunikative } \\
\text { Aktivitäten }\end{array}$ & .73 & 4.1 & 1.4 \\
\hline $\begin{array}{l}\text { Sich über neue Vorgehensweisen im Unterricht } \\
\text { austauschen. }\end{array}$ & & .59 & 3.6 & 1.4 \\
\hline
\end{tabular}

a standardisierte Faktorladung CFA: $\mathrm{N}=805$, chi-square $=58.32 ; \mathrm{df} 31, \mathrm{cmin} / \mathrm{df}=1.881, \mathrm{p}$-value $=.00$, pclose $=.99, \mathrm{CFI}=.99, \mathrm{TLI}=.98, \mathrm{RMSEA}=.033, \mathrm{SRMR}=.025^{1}$.

b Mittelwert/Skalierung: fast nie/nie=1; ca. 1xpro Jahr=2; ca. mehrmals pro Jahr=3; ca. 1x pro Monat=4; ca. mehrmals pro Monat=5, ca. 1x pro Woche=6, ca. mehrmals pro Woche=7.

Aus Tabelle 2 geht hervor, dass am häufigsten fachbezogene Bücher oder Zeitschriften gelesen werden, im Schnitt zwischen ein- und mehrmals pro Monat, während die pädagogisch-didaktische Lektüre zu den weit selteneren Aktivitäten gehört. Mit durchschnittlich einmal im Monat werden vergleichsweise häufig im kollegialen Austausch Überlegungen zur besseren Förderung einzelner Schülerinnen und Schüler angestellt. In einer mittleren Frequenz (einmal pro Monat 
und mehrmals pro Jahr) werden verbindliche Absprachen über den Unterricht getroffen, neue Vorgehensweisen besprochen, Schüler/innen-Rückmeldungen eingeholt; Es wird Unterrichtsmaterial von Kolleginnen und Kollegen übernommen oder ihnen Unterstützung angeboten. Mit durchschnittlich mehrmals pro Jahr eher selten, wird gemeinsam mit Kolleginnen und Kollegen der Unterricht vorbereitet, werden neue Unterrichtsformen erprobt und pädagogisch didaktische Bücher oder Zeitschriften gelesen.

\section{Modifiziertes Modell}

Aufgrund einer ersten Analyse der Daten (Kunz Heim et al., 2007) zeigte sich, dass der postulierte Faktor wahrgenommene Führung keinen wesentlichen Beitrag zur Erklärung der beiden Komponenten des Weiterlernens von Lehrpersonen leistet: Innerhalb der wahrgenommenen Führung wies die Dimension zielgerichtete Führung eine sehr geringe $(r<.10)$ Korrelation mit der pragmatischen Form des informellen Weiterlernens auf. Die Dimension Förderung korrelierte ebenfalls sehr gering positiv mit der Dimension pragmatisches Weiterlernen sowie mit der Dimension kommunikatives Weiterlernen. Alle weiteren Führungsdimensionen zeigten keine signifikanten Zusammenhänge mit dem Weiterlernen von Lehrpersonen (ebd., S. 41).

Es stellte sich auch heraus, dass praktisch keine Dimension der postulierten Prädiktorvariablen einen Zusammenhang mit der institutionellen Komponente des Weiterlernens aufwies. Einzig die Dimension zielgerichtete Führung zeigte eine sehr geringe Korrelation mit dem institutionellen Weiterlernen.

Bezüglich des institutionellen Weiterlernens spielte hauptsächlich die Kantonszugehörigkeit sowie Aspekte der spezifischen Anstellungs- bzw. Berufssituation eine Rolle: Lehrpersonen aus dem Kanton Thurgau ${ }^{2}$, die mit einem Pensum von über $40 \%$ angestellt sind, mehr als ein Dienstjahr aufweisen und an der Schule Spezialfunktionen wahrnehmen, vermeldeten mehr institutionelle Weiterbildung. Die genannten Faktoren erklären zusammen rund 24\% der Varianz innerhalb des institutionellen Weiterlernens (Kunz Heim et al., 2007, S. 35).

Im Gegensatz zum institutionellen Weiterlernen ist der Einfluss soziodemografischer und berufsbezogener Faktoren auf die Wahrscheinlichkeit des Auftretens des informellen Weiterlernens geringer: Lediglich rund 6\% der Varianz wird durch entsprechende Einflüsse erklärt, wobei die Anzahl spezieller Funktionen und das Pensum am meisten Gewicht haben (ebd., S. 37). Das institutionelle und das informelle Weiterlernen wiesen keinen Zusammenhang mehr auf, nachdem das Pensum und die Anzahl Spezialfunktionen kontrolliert worden sind (ebd., S. 38). 
Als geeignete Prädiktoren des informellen Weiterlernens erwiesen sich die Ausprägung der Praxisgemeinschaft sowie bestimmte Bereiche des Kompetenzselbstkonzeptes.

Das in Abbildung 1 vorgestellte heuristische Modell musste somit stark modifiziert werden: Wie erwähnt musste aufgrund der bivariaten Analyse die Annahme verworfen werden, wonach mit den postulierten Prädiktorvariablen die Verbreitung des institutionellen Weiterlernens erklärt werden kann. Ebenso musste die Prädiktorvariable wahrgenommene Führung verworfen werden. Im modifizierten Modell (Abbildung 2) wird die Erwartung an das Zusammenwirken der relevanten verbleibenden Faktoren dargestellt, die sich gegenüber dem heuristischen Modell in Abbildung 1 nicht verändert hat.

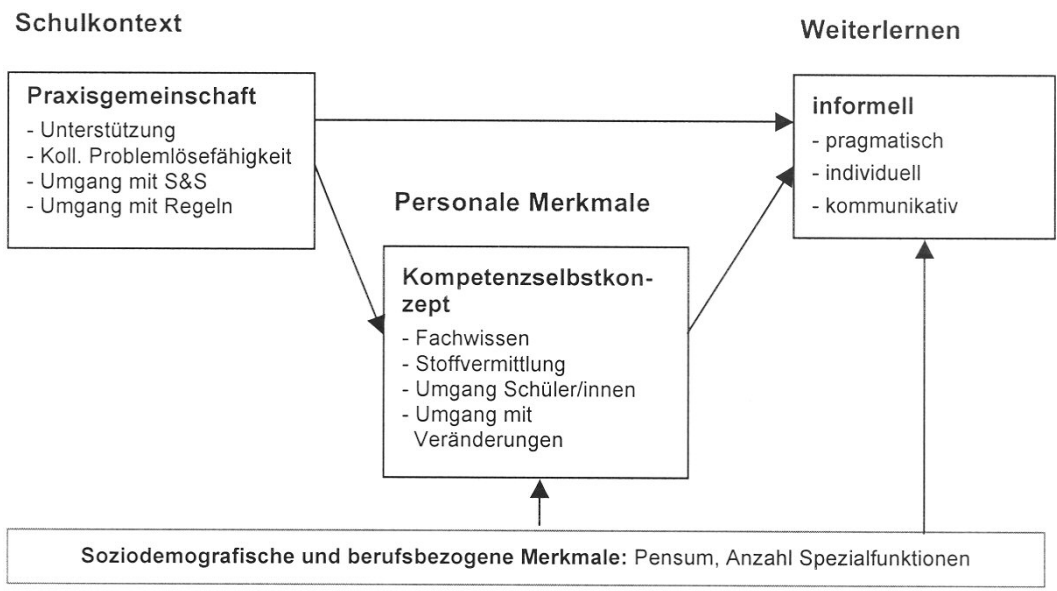

\section{Abbildung 2: Modifiziertes Modell}

Allerdings enthält das modifizierte Modell nicht mehr alle der ursprünglich in die Untersuchung aufgenommenen Dimensionen der Praxisgemeinschaft und des Kompetenzselbstkonzeptes. In der konfirmatorischen Faktorenanalyse (CFA) der Praxisgemeinschaft zeigte sich, dass das Ausmass wahrgenommener Konflikte und Spaltungen innerhalb des Kollegiums (Harmonie/Klima) einen Bereich darstellt, der für sich alleine steht und nicht dem Konstrukt der Praxisgemeinschaft subsumiert werden sollte ${ }^{3}$.

Bezüglich des Kompetenzselbstkonzeptes sind die beiden Dimensionen soziale Kompetenz im Umgang mit Eltern und soziale Kompetenz im Umgang mit Vorgesetzten nicht in das modifizierte Modell aufgenommen worden. Was die soziale Kompetenz im Umgang mit Eltern betrifft, so war die Fallzahl zu niedrig, 
weil die Fragen nur von Klassenlehrpersonen, nicht aber von Fachlehrpersonen ohne Elternkontakt beantwortet werden konnten. Die soziale Kompetenz im Umgang mit Vorgesetzten wurde nicht aufgenommen, weil die entsprechenden Fragen während des Erhebungszeitraums einen grossen Bedeutungswandel erfuhren und daher von Lehrpersonen in geleiteten Schulen im Vergleich zu solchen in ungeleiteten Schulen auch anders interpretiert wurden.

\section{Die Verbreitung des informellen Weiterlernens als Funk- tion der Praxisgemeinschaft und des Kompetenzselbstkon- zeptes}

Das modifizierte Modell konnte bestätigt werden. Aus Abbildung 3 ist ersichtlich, dass ein hoher Wert des Prädiktors Praxisgemeinschaft bedeutet, dass Lehrpersonen im Kollegium mehr Unterstützung und Ansporn, eine gute Problemlösefähigkeit, einen konsequenten Umgang mit Regeln und einen anspruchsund respektvollen Umgang mit Schülerinnen und Schülern wahrnehmen.

Die Ausprägung der Praxisgemeinschaft hat einen direkten positiven Einfluss auf zwei Dimensionen des informellen Weiterlernens und zwar auf das pragmatische $(.10, \mathrm{p}<.03)$ und das kommunikative $(.11, \mathrm{p}<.02)$ Weiterlernen. Sehr geringe indirekte Effekte der Praxisgemeinschaft zeigen sich auf das individuelle $(.05, \mathrm{p}<.01)$ und wiederum auf das kommunikative $(.09, \mathrm{p}<.01)$ Weiterlernen.

Etwas stärker sind allerdings die direkten Effekte der Kompetenzselbstkonzepte auf die individuellen und kommunikativen Formen des Weiterlernens: Je stärker das Selbstkonzept in den Kompetenzbereichen Fachwissen $(.21, \mathrm{p}<.01)$ und Umgang mit Veränderungen $(.13, \mathrm{p}<.01)$ ist, desto wahrscheinlicher sind individuelle Formen des informellen Weiterlernens. Je höher das Kompetenzselbskonzept in den Bereichen Stoffvermittlung (.17, p<.01) sowie im Umgang mit Schülerinnen $(.22, \mathrm{p}<.01)$ eingeschätzt wird, desto eher werden kommunikative Formen des informellen Weiterlernens gepflegt. 


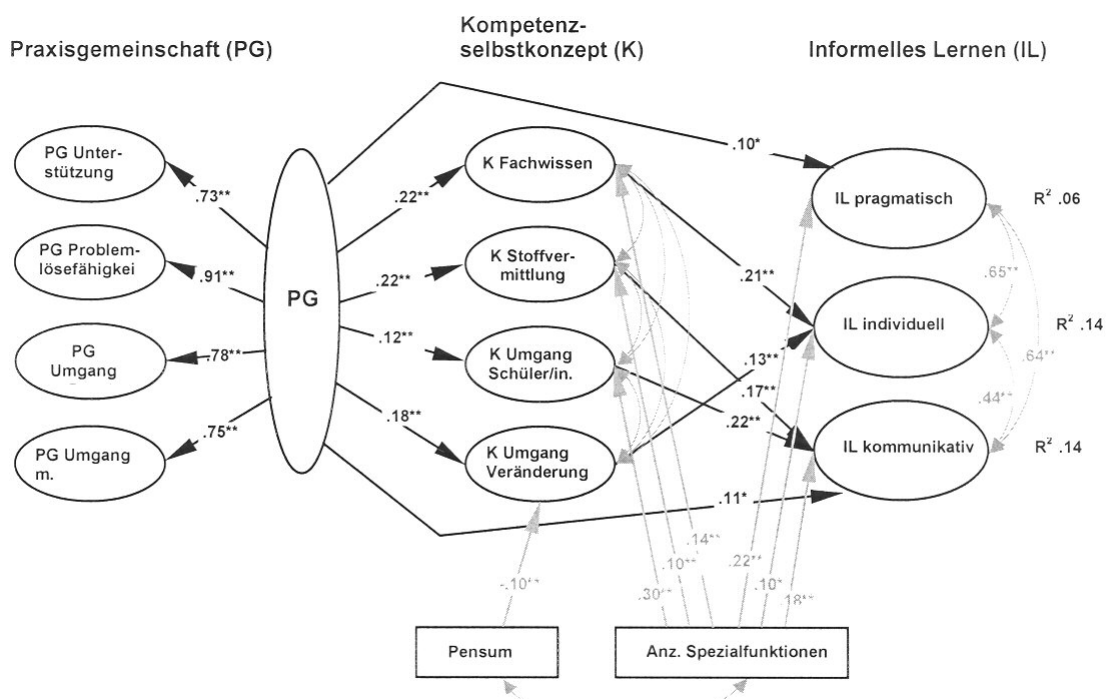

Abbildung. 3: Strukturgleichungsmodell Informelles Weiterlernen (modifiziertes Modell)

${ }^{*}=\mathrm{p}<.05 /{ }^{* *}=\mathrm{p}<.01$, die Korrelationen der Kompetenzbereiche sind in der Fussnote tabellarisch aufgeführt $^{4}$

Stand. estimates, $\mathrm{N}=763$, chi-square 1139.66 , $\mathrm{df} 636, \mathrm{cmin} / \mathrm{df}=1.792$;

$\mathrm{p}$-value $=00$, pclose $=1, \mathrm{CFI}=.94, \mathrm{TLI}=.95, \mathrm{RMSEA}=.032, \mathrm{SRMR}=.0425$

Zur Aufklärung der Varianzen $\left(\mathrm{R}^{2}\right)$ trägt die Anzahl Spezialfunktionen als Aspekt der beruflichen Situation am meisten bei: Die Anzahl schulischer Spezialfunktionen zeigt direkte Effekte auf alle drei Dimensionen des informellen Lernens (pragmatisch: .22, $\mathrm{p}<.01$; individuell: .10, $\mathrm{p}<.01$; und kommunikativ: $.18, \mathrm{p}<.01)$. Zudem wird die Vermutung bestätigt, dass der Effekt der Anzahl Spezialfunktionen ebenfalls durch Kompetenzselbstkonzept mediiert wird: So sagt eine hohe Anzahl Spezialfunktionen ein höheres Kompetenzselbstkonzept in den Bereichen Fachwissen (.14, $\mathrm{p}<.01)$, Stoffvermittlung $(.10, \mathrm{p}<.01)$ und Umgang mit Schülerinnen und Schülern $(.30, \mathrm{p}<.01)$ voraus, was geringe indirekte Effekte der Anzahl Spezialfunktionen auf die kommunikative (.06, $\mathrm{p}<.01)$ sowie auf die individuelle (.03, $\mathrm{p}<.01)$ Form informellen Lernens erzeugt.

Die zweite Kontextvariable Pensum hängt mit der Anzahl Spezialfunktionen zusammen $(.45, \mathrm{p}<.01)$, d.h. je höher das Pensum, desto wahrscheinlicher ist auch eine grössere Anzahl spezieller schulischer Funktionen. Ein schwacher indirekter Einfluss des Pensums wird im Bereich des Kompetenzselbstkonzeptes im Um- 
gang mit Veränderungen deutlich: Je niedriger das Pensum, desto höher wird die eigene Kompetenz im Umgang mit Veränderungen wahrgenommen (-.10, $\mathrm{p}<.01)$.

\section{Diskussion}

In der vorliegenden Studie wurden Faktoren postuliert, welche die Verbreitung des informellen und des institutionellen Weiterlernens von Lehrpersonen beeinflussen. Entgegen den Annahmen konnte die institutionelle Komponente des Weiterlernens mit den ausgewählten Faktoren nicht erklärt werden. Die informelle Komponente des Weiterlernens hingegen wurde durch die Ausprägung der Praxisgemeinschaft und des Kompetenzselbstkonzeptes auch dann beeinflusst, wenn das Engagement in schulischen Spezialfunktionen und das Pensum der Lehrpersonen ins Modell einbezogen wurden. Der Anteil der aufgeklärten Varianz blieb insgesamt allerdings eher gering.

Was die Verbreitung des institutionellen Weiterlernens betrifft, so haben sich die Zahlen im Vergleich zur Studie von Landert (1999) verschlechtert. Waren es damals wie erwähnt 40\% der Lehrpersonen, die das Weiterbildungsangebot mit weniger als 5 Tagen pro Jahr nutzten, sind es in der vorliegenden Studie 64\%, wobei berücksichtigt werden muss, dass der Anteil der Lehrpersonen in Teilzeitpensen in dieser Studie 55\% beträgt, während er bei Landert lediglich 42\% ausmachte (ebd., S. 42).

Die Verbreitung des informellen Weiterlernens ist mit derjenigen in der Untersuchung von Kwakman (2003) insofern vergleichbar, als dass Tätigkeiten, die in der vorliegenden Studie vergleichsweise häufig genannt werden, auch in der Studie von Kwakman häufiger angegeben wurden und umgekehrt (Kunz Heim et al., 2007, S. 48). Zur konkreten Häufigkeit entsprechender Aktivitäten gibt es bisher hingegen noch keine validen Vergleichszahlen.

Was die postulierten Faktoren des Schulkontextes betrifft, so zeigt die wahrgenommene Führung weder Einfluss auf die institutionelle noch auf die informelle Komponente des Weiterlernens. Dies steht im Widerspruch zu vergleichbaren Studien aus dem angloamerikanischen Raum, in denen die Effekte der Führung auf das Weiterlernen nachgewiesen wurden (Richardson \& Placier, 2001; Rosenholtz et al., 1986). Eine mögliche Interpretation dieser Differenz könnte darin begründet sein, dass in den beiden Kantonen, in denen die Befragung durchgeführt wurde, die Schulleitungen erst seit dem Schuljahr 2002/2003 schrittweise eingeführt worden sind und zum Befragungszeitpunkt höchstens drei Jahre, an einigen Schulen noch gar nicht implementiert waren und die Zeitspanne zu kurz war, als dass die Schulleitungen ihre Wirkung hätten voll entfal- 
ten können. In diese Richtung deutet zumindest der Befund, dass die Bereiche Zielführung, Innovationsbereitschaft und Förderung signifikant besser eingeschätzt werden, je weiter der Implementierungsprozess des Schulleitungsmodells fortgeschritten ist (Kunz Heim et al., 2007, S. 22).

Wie im modifizierten Modell angenommen, wird das informelle Weiterlernen durch Faktoren des Schulkontextes und durch personale Faktoren der Lehrpersonen beeinflusst, wobei folgende Unterschiede festzustellen sind:

- Das pragmatische Weiterlernen wird durch die Ausprägung der Praxisgemeinschaft direkt beeinflusst, jedoch, entgegen den Erwartungen, nicht durch das Kompetenzselbstkonzept (KSK).

- Das individuelle Weiterlernen wird durch das Kompetenzselbstkonzept in den Bereichen Fachwissen und Veränderung direkt und durch die Ausprägung der Praxisgemeinschaft sehr gering indirekt beeinflusst (mediiert durch das KSK).

- Das kommunikative Weiterlernen wird durch das Kompetenzselbstkonzept in den Bereichen Stoffvermittlung und Umgang mit Schülerinnen und Schülern direkt beeinflusst. Zudem wird es durch die Ausprägung der Praxisgemeinschaft sowohl direkt als auch in geringem Masse indirekt (mediiert durch das KSK) beeinflusst.

Berufsbezogene Merkmale haben einen hohen Einfluss auf das informelle Weiterlernen. So lässt sich das informelle Weiterlernen insgesamt am besten durch die Anzahl schulischer Spezialfunktionen voraussagen, die eine Lehrperson innehat. Der entsprechende Effekt entsteht hauptsächlich direkt, sowie in geringem Masse mediiert durch das Kompetenzselbstkonzept (KSK) in den Bereichen Fachwissen, Stoffvermittlung sowie Umgang mit Schülerinnen und Schülern. Eine hohe Anzahl schulischer Spezialfunktionen geht zudem einher mit einer erhöhten Wahrscheinlichkeit für ein höheres Pensum.

Insgesamt stehen die Daten nicht im Widerspruch zum modifizierten Modell, womit die darin enthaltenen Annahmen über das Zusammenwirken der postulierten Faktoren beibehalten werden können: Lehrpersonen, die in gut funktionierenden Praxisgemeinschaften arbeiten, entwickeln ein höheres Kompetenzselbstkonzept, das sie motiviert individuell weiterzulernen oder in einen eher unverbindlichen kommunikativen Austausch mit Kolleginnen und Kollegen zu treten.

Lehrpersonen, die in gut funktionierenden Praxisgemeinschaften arbeiten, sind eher motiviert, Lernaktivitäten im pragmatischen und kommunikativen Bereich auszuführen wo sie sich über Unterricht austauschen, aber auch konkrete Unterlagen erarbeiten oder Absprachen treffen, die von ihnen unter Umständen Kompromisse in Bezug auf ihre gewohnten Herangehensweisen an die Unterrichtsgestaltung verlangen.

Die Bedeutung des Kompetenzselbstkonzeptes stimmt überein mit der Sicht- 
weise von Pajares und Schunk (2001) sowie mit früheren Befunden, wonach personale Faktoren einen bedeutenden Einfluss auf Lernaktivitäten von Lehrpersonen haben (Kwakman, 2003; Smylie, 1998).

Beachtung verdient die Bedeutung der Anzahl der Spezialfunktionen, welche Lehrpersonen im Rahmen des Schulalltages übernehmen. Diese hat sowohl auf drei der vier Dimensionen des Kompetenzselbstkonzepts als auch auf alle drei Dimensionen des informellen Weiterlernens positive Effekte. Es zeigt sich, dass sich die zusätzliche Verantwortung, die Lehrpersonen mit den Spezialfunktionen übernehmen, positiv auf die genannten Faktoren auswirkt.

\section{Einschränkungen der Aussagekraft und weiterführende Fragestellungen}

Da es sich um eine Querschnittuntersuchung handelt, müssen auch bei Strukturgleichungsmodellen Vorbehalte in Bezug auf die Richtung der Kausalität gemacht werden. Sicherheit über die hier dargestellten Richtungen können erst durch Längschnitte gewonnen werden. Ein weiterer Vorbehalt ergibt sich aus einem kleinen Teil der Operationalisierungen. So weist die Subdimensionen Unterstützung des Konstruktes Praxisgemeinschaft in der Itemformulierung gewisse Ähnlichkeiten mit der Dimension des kommunikativen Weiterlernens auf. Mit der vorliegenden Untersuchung ist zwar ein weiterer Schritt in Bezug auf die Operationalisierung des informellen Weiterlernens gemacht worden, indem die Faktorenstruktur der Skalen von Kwakman (2003) bestätigt werden konnte; Die Operationalisierung bleibt aber auf einem pauschalen Niveau, so dass diesbezüglich weitere Anstrengungen unternommen werden müssen.

Der Umstand, wonach die verschiedenen Dimensionen des informellen Weiterlernens durch die postulierten Faktoren unterschiedlich beeinflusst werden, wirft Fragen nach dem Charakter der unterschiedlichen Lernaktivitäten auf, es fragt sich aber auch, ob es unter Lehrpersonen verschiedene Typen mit unterschiedlichen Präferenzen bezüglich des informellen Weiterlernens gibt.

Wie erwähnt kann das Weiterlernen von Lehrpersonen durch die in der vorliegenden Studie ausgewählten Faktoren generell nur bedingt erklärt werden. Die Suche nach weiteren Einflussfaktoren ist im Hinblick auf die schulische Qualitätsdiskussion weiterhin von Bedeutung.

\section{Konsequenzen für die Praxis}

Wie eingangs erwähnt, hat das Weiterlernen von Lehrpersonen eine hohe Bedeutung für die Umsetzung von Reformbestrebungen sowie für die Erhaltung und Erhöhung der Unterrichts- und Schulqualität. Die vorliegende Studie liefert Hinweise, wonach sich die Anstrengungen für den Aufbau einer Praxisgemeinschaft (Professionellen Lerngemeinschaft) lohnen, in der sich Lehrpersonen gegenseitig unterstützen und anspornen, eine hohe Problemlösefähigkeit, einen konsequenten Umgang mit Regeln und einen anspruchs- und respektvollen 
Umgang mit Schülerinnen und Schülern pflegen. Dies kann zu einem erhöhten Kompetenzselbstkonzept und zu einer erhöhten Lernaktivität der Lehrpersonen im informellen Bereich führen.

Aufgrund der positiven Effekte der Anzahl Spezialfunktionen empfiehlt es sich auch, eine solche funktionale Differenzierung innerhalb der Schulen im $\mathrm{Zu}$ sammenhang mit dem Aufbau von Professionellen Lerngemeinschaften auszubauen. So kann das Engagement und die Erfahrungen der Lehrpersonen, welche bereit sind, Verantwortung zu übernehmen, entsprechend den Empfehlungen von Bolam (2008), für diesen Zweck genutzt und somit verstärkt werden.

Darüber hinaus muss eine ausreichende Nutzung der Angebote des institutionellen Weiterlernens sichergestellt werden. Auch wenn Lehrpersonen intensiv voneinander durch Austausch und Zusammenarbeit weiterlernen, kann auf Impulse von aussen nicht verzichtet werden (King, 2004). Besondere Beachtung sollten dabei auch Lehrpersonen erfahren, die in Kleinpensen $(<40 \%)$ angestellt sind. Aufgrund der Ergebnisse der Studie stellt sich die Frage, welches Ausmass an Kleinpensen eine Schulgemeinschaft verkraften kann bez. wie diese Gruppe von Lehrpersonen spezifisch unterstützt werden sollte.

\section{Anmerkungen}

1 Die angegebenen Fitwerte beziehen sich auf ein gegenüber dem Grundlagenbericht (Kunz Heim et al., 2007) leicht verändertes Messmodell: In der Subdimension pragmatisches informelles Weiterlernen wurde das folgende Item entfernt: «Prüfungen austauschen, um die Leistungen unterschiedlicher Klassen zu vergleichen». Im Laufe vertiefter Analysen hatte sich gezeigt, dass diese Aktivität von unterschiedlicher Bedeutung ist, je nach dem ob es sich bei den Antwortenden um Fach- oder Klassenlehrpersonen handelte.

2 Lehrpersonen aus dem Kanton Thurgau hatten schon in früheren Studien überdurchschnittlich viele Weiterbildungsaktivitäten verzeichnet (Kunz Heim et al., 2007, S. 46). Zudem sei in Erinnerung gerufen, dass auch eine mögliche Verzerrung der Thurgauer Stichprobe einen entsprechenden Effekt begünstigt (Tabelle1).

3 Überdies zeigten sich auch keine Zusammenhänge zwischen dem Klima im Kollegium und dem informellen Weiterlernen der Lehrpersonen (Kunz Heim et al., 2007, S. 42), die allenfalls einen Einbezug ins Modell nahegelegt hätten.

4

\begin{tabular}{|l|l|l|l|}
\hline K Stoffvermittlung & $<->$ & K Umg. mit Veränderungen & $.46^{* *}$ \\
\hline K Stoffvermittlung & $<->$ & K Umg. mit Schüler/innen & $.45^{* *}$ \\
\hline K Fachwissen & $<-->$ & K Stoffvermittlung & $.44^{* *}$ \\
\hline K Fachwissen & $<->$ & K Umg. mit Veränderungen & $.36^{* *}$ \\
\hline K Umg. mit Schüler/innen & $<-->$ & K Umg. mit Veränderungen & $.31^{* *}$ \\
\hline K Fachwissen & $<-->$ & K Umg. mit Schüler/innen & $.21^{* *}$ \\
\hline
\end{tabular}

5 Bei der Interpretation der Fitwerte der konfirmatorischen Faktorenanalyse und des Strukturgleichungsmodells ist zu berücksichtigen, dass der p-Wert des $\mathrm{Chi}^{2}$-Testes bei grossen Stichproben sehr schnell signifikant wird (Kline, 1998). Es sind daher weitere, geeignete Indikatoren aufgeführt. Neben der $\mathrm{Chi}^{2}$-Statistik wurde insbesondere der RMSEA (root mean square error of approximation und das dazugehörige PCLOSE, vgl. Brown \& Cudeck, 1993), CFI (comprative fit index, vgl. Bentler, 1990), TLI (Tucker-Lewis-Index, vgl. Tuker \& Lewis, 1973), SRMR (Standardized Root Mean Square Residual, vgl. Hu \& 
Bentler, 1999) berücksichtigt. Unabhängig vom p-Wert des $\mathrm{Chi}^{2}$-Tests wurden die Modelle unter folgenden Bedingungen als passend akzeptiert: Chi $2 / \mathrm{df}<3$; RMSEA < .05; PCLOSE>.95; TLI > .95 und SRMR <.05 (vgl. Hu \& Bentler, 1999; Kline, 1998).

\section{Literaturverzeichnis}

Bolam, R. (2008). Professional learning communities and teachers' professional development. In D. Johnson \& R. Maclean (Ed.), Teaching: Professionalization, development and leadership (pp. 159-179). London: Springer.

Bonsen, M. \& Rolff, H. G. (2006). Professionelle Lerngemeinschaften von Lehrerinnen und Lehrern. Zeitschrift für Pädagogik, 52 (2), 167-184.

Bonsen, M., Van der Gathen, J., Iglhaut, C. \& Pfeiffer, H. (2002). Die Wirksamkeit von Schulleitung. Empirische Annäherungen an ein Gesamtmodell schulischen Leitungshandelns. Weinheim: Juventa.

Brown, M. W. \& Cudeck, R. (1993). Alternative ways of assessing model fit. In K. A. Bollen and J. S. Long (Ed.), Testing structural equation models (pp. 136-163). Newbury Park: Sage.

Brüsemeister, T. \& Eubel, K. D. (2003). Facetten schulischer Modernisierung. In T. Brüsemeister und K.-D. Eubel (Hrsg.), Zur Modernisierung der Schule. Leitideen - Konzepte Akteure. Ein Überblick (S. 15-33). Bielefeld: Transcript.

Center for Research on the Context of Teaching (2001). BASRC Teacher Survey Scales 2001. Retrieved 15.12.2009 from http://www.stanford.edu/group/CRC/survey_instruments.htm

Center for Research on the Context of Teaching (2002). BASRC Teacher Survey Scales 2002. Retrieved 15.12.2009 from http://www.stanford.edu/group/CRC/survey_instruments.htm

Clarke, D. \& Hollingsworth, H. (2002). Elaborating a model of teacher professional growth. Teaching and Teacher Education, 18 (8), 947-967.

Fend, H. (1998). Qualität im Bildungswesen. Schulforschung zu Systembedingungen, Schulprofilen und Lehrerleistung. Weinheim: Juventa.

Fichten, W. (2007). Kooperative Unterrichtsentwicklung. Ergebnisse und Prozesserfahrungen eines Entwicklungsprojekts. Pädagogik, 59 (10), 38-43.

Ghisla, G. (1999). Lehrplanmetaphern und pädagogischer Common Sense. In R. Künzli, K. Bähr, A. V. Fries, G. Ghisla, M. Rosenmund \& G. Seliner-Müller (Hrsg.), Lehrplanarbeit: über den Nutzen von Lehrplänen für die Schule und ihre Entwicklung. Nationales Forschungsprogramm 33, Wirksamkeit unserer Bildungssysteme (S. 124-143). Chur: Rüegger.

Hargreaves, A. (1997). Cultures of teaching and educational change. In B. J. Biddle, T. L. Good \& I. F. Goodson (Ed.), International Handbook of Teachers and Teaching, (Vol. 2, pp. 1297-1319). London: Kluwer Academic Publishers.

Hu, L. \& Bentler, P. M. (1999). Cutoff criteria for fit indexes in covariance structure analysis: Conventional criteria versus new alternatives. Structural Equation Modeling, 6, 1-55.

King, M. B. (2004). School- and district-level leadership for teacher workforce development: Enhancing teacher learning and capacitiy. In M. A. Smylie and D. Miretzky (Ed.), Developing the teacher workforce. 103rd Yearbook of the National Society for the Study of Education (pp. 303-325). University of Chicago Press.

Kline, R. B. (1998). Principles and practice of structural equation modelling. New York: Guilford Press.

Krüger, M. (1994). Sekseverschillen in schoolleidershap. Alphen aan de Rijn.

Kunz Heim, D. (2002). Qualität durch Qualifizierung. Lehrerbeurteilung als Instrument zur Förderung von Qualität im Unterricht. Weinheim: Juventa.

Kunz Heim, D., Trachsler, E., Rindlisbacher, S. \& Nido, M. (2007). Schulen als Lernumgebungen für Lehrerinnen und Lehrer. Zusammenhänge zwischen Schulkontext, persönlichen 
Merkmalen und dem Weiterlernen von Lehrpersonen. (Forschungsbericht 06 / Oktober 07). Pädagogische Hochschule Thurgau, Kreuzlingen (Hrsg.) in Zusammenarbeit mit Institut Forschung und Entwicklung der Fachhochschule Nordwestschweiz, Aarau.

Kwakman, K. (2003). Factors affecting teacher's participation in professional learning activities. Teaching \& Teacher Education, 19, 149-170.

Landert, C. (1999). Lehrerweiterbildung in der Schweiz. Ergebnisse der Evaluation von ausgewählten Weiterbildungssystemen und Entwicklungslinien für eine wirksame Personalentwicklung in den Schulen. Nationales Forschungsprogramm 33, Wirksamkeit unserer Bildungssysteme. Chur: Rüegger.

McLaughlin, M. W. (1993). What matters most in teachers' workplace context? In J. W. Little \& M. W. McLaughlin (Ed.), Teachers' work. Individual's, colleagues, contexts (pp. 79-103). New York: Teachers College Press.

McLaughlin, M. W. (1998). Listening and learning from the field: Tales of policy implementation and situated practice. In A. Hargreaves, A. Lieberman, M. Fullan \& D. Hopkins (Ed.), International Handbook of Educational Change (pp. 70-84). Dordrecht: Kluwer Academic Publishers.

Mutzeck, W. (1992). Verwirklichung von Handlungsabsichten im Berufsalltag. Rekonstruktion Subjektiver Theorien. Zeitschrift für Pädagogische Psychologie, 6 (1), 1-15.

Pajares, F. \& Schunk, D. H. (2001). Self-beliefs and school success: Self efficacy, self-concept, and school achievement. In R. J. Riding \& S. G. Rayner (Ed.), Self Perception. International perspectives on individual differences (pp. 239-265). Westport: Ablex Publishing.

Richardson, V. \& Placier, P. (2001). Teacher change. In V. Richardson (Ed.), Handbook of research on teaching (4th ed., pp. 905-947). Washington, D.C.: American Educational Research Association.

Rolff, H. G. (1988). Ansätze zu einer Theorie der Schulentwicklung. Werkheft 28. Universität Dortmund, Institut für Schulentwicklungsforschung.

Rolff, H. G. (1995). Wandel durch Selbstorganisation. Theoretische Grundlagen und praktische Hinweise für eine bessere Schule. Weinheim: Juventa.

Rosenholtz, S. J. (1991). Teachers' workplace: The social organisation of schools (2nd ed.). New York: Teachers College, Columbia University.

Rosenholtz, S. J., Bassler, O. \& Hoover-Dempsey, K. (1986). Organizational conditions of teacher learning. Teaching \& Teacher Education, 2 (2), 91-104.

Rudduck, J. (1988). The ownership of change as a basis for teachers' professional learning. In J. Calderhead (Ed.), Teachers' professional learning (pp. 205-222). London: Falmer Press.

Rüegg, S. (2000). Weiterbildung und Schulentwicklung: eine empirische Studie zur Zusammenarbeit von Lehrerinnen und Lehrern. Bern: Peter Lang.

Skaalvik, E. M. (1997). Issues in research on self-concept. In M. Maehr \& P. R. Pintrich (Ed.), Advances in motivation and achievement (Vol. 10, pp. 51-97). New York: JAI Press.

Smylie, M.A. (1988). The enhancement of function of staff development: Organizational and psychological antecendents to individual teacher change. American Educational Research Journal, 25 (1), 1-30.

Tabachnick, B. G. \& Fidell, L. S. (1989). Using Multivariate Statistics. New York: Harper \& Row.

Tucker, L. R. \& Lewis, C. (1973). A reliability coefficient for maximum likelihood factor analysis. Psychometrika, 38, 1-10.

Ulber, D. (1998). ODAS Survey-Feedback-Instrument zur Organisationsdiagnose an Schulen. Zugriff am 15.12.2009 unter http://www.zpid.de/index.php?wahl=products\&uwahl=frei\&uuwahl=testarchiveintro\#o 
Schlagworte: Professionelle Entwicklung von Lehrpersonen, Weiterbildung von Lehrpersonen, informelles Weiterlernen von Lehrpersonen, institutionelles Weiterlernen von Lehrpersonen, Professionelle Lerngemeinschaften, Schulentwicklung, Selbstkonzept, Selbstwirksamkeitsüberzeugung

\section{La formation continue des enseignant-e-s: effets de la communauté professionnelle et du concept de soi en lien avec les compétences}

Résumé

La formation continue des enseignant-e-s est une condition importante de la qualité de l'école et de l'enseignement mais aussi de la mise en œuvre des réformes. La présente étude s'est intéressée à l'influence du contexte scolaire et des facteurs personnels des enseignant-e-s sur l'implication dans la formation continue institutionnelle et informelle. Un modèle a été mis à l'épreuve sur la base des données recueillies auprès d'un échantillon représentatif d'enseignant-e-s du secondaire I dans les cantons d'Argovie et de Thurgovie. Les résultats de l'étude ont mis en évidence l'importance de la communauté de pratiques des enseignant-e-s de l'école, du concept de soi en lien avec les propres compétences et le nombre de fonctions spéciales qu'ils exercent. Contrairement aux attentes, les facteurs postulés n'ont pas permis d'expliquer la formation continue institutionnelle.

Mots clés: Formation continue des enseignant-e-s, qualité de l'école, communauté des pratiques des enseignant-e-s, concept de soi des compétences

\section{La formazione continua delle/gli insegnanti: effetti della comunità di pratica e della concezione personale di competenza}

\section{Riassunto}

La formazione continua delle/gli insegnanti è una condizione importante della qualità della scuola e dell'insegnamento, ma anche dell'implementazione delle riforme. Il presente studio si è interessato all'influenza del contesto scolare e dei fattori personali delle/gli insegnanti sulla partecipazione alla formazione continua istituzionale o informale. Un modello è stato verificato sulla base dei dati raccolti presso un campione rappresentativo d'insegnanti del secondario I nei cantoni di Argovia e di Turgovia. I risultati dello studio hanno messo in evidenza l'importanza, per la partecipazione alla formazione continua informale, della comunità di pratica delle/gli insegnanti, della concezione personale di competenza e del numero di funzioni speciali che gli insegnanti esercitano. Contrariamente 
alle attese, i fattori postulati non hanno permesso di spiegare la formazione continua istituzionale.

Parole chiave: Formazione continua delle/gli insegnanti, qualità della scuola, comunità di pratica, auto-valutazione delle competenze

\section{The frequency of continuing education of teachers: Effects of the professional teaching community and the sense of self (in relation to qualifications)}

\footnotetext{
Abstract

The continuing education of teachers is an important requirement for the quality of the school and the teaching, but also for the implementation of change. This study is interested in the influence of the educational context and personal characteristics of teachers on their involvement in institutional and informal continuing education programs. A model based on collected data from a representative teacher sample at the lower secondary level in the cantons of Aargau and Thurgau was tested. Results showed the importance of the professional teaching community, a sense of self, along with their own competencies as well as a number of specialized teacher duties. In contrast to the original assumptions, the postulated factors were not able to explain institutionalized, continuing teacher education.
}

Key words: Teacher, change, teacher development, institutional teacher learning, informal teacher learning, professional learning community, school effectiveness, self-concept, self-efficacy 